\title{
Diffraction efficiency of low-resolution Fresnel encoded lenses
}

\author{
E. Carcolé, J. Campos, I. Juvells, and S. Bosch
}

\begin{abstract}
A mathematical model to describe the behavior of low-resolution Fresnel lenses encoded in any low-resolution device (e.g., a spatial light modulator) is developed. From this model the diffraction efficiency is calculated in terms of all the parameters that characterize these lenses.

Key words: Fresnel lens, zone plate, diffraction, spatial light modulator, array illuminator.
\end{abstract}

\section{Introduction}

One of the most promising applications of pixelated spatial light modulators (SLM's) is their use as variable focal-length lenses in optical setups. ${ }^{1}$ This involves the low-resolution codification of a quadratic wave. The effects of this process on lens performance have been described recently with a mathematical model developed by Carcolé et al. ${ }^{2}$ With this model an expression for the light-amplitude distribution for all focal regions can be established. This expression is a function of several adimensional parameters that depend on the characteristics of the low-resolution device and the focal length encoded. This implies a dependence of the shape of the distribution on the focal length.

The most important difficulty of a low-resolution Fresnel encoded lens (LRFEL) is its multifocusing property, which involves an important loss of image quality and diffraction efficiency. On the other hand, this is an advantage if the LRFEL is used as an array illuminator. ${ }^{3}$ The aim of this paper is to perform a rigorous calculation of the diffraction efficiency of a LRFEL making use of the model. In Section 2 the main results of Ref. 2 are explained in relation to our development. The diffraction efficiency is obtained for the $(0,0)$ order in Section 3 and for the $(k, l)$ order in Section 4. In Section 5 we comment on the

E. Carcolé, I. Juvells, and S. Bosch are with the Departament de Física Aplicada i Electrònica, Laboratori d'Optica, Diagonal 647, Barcelona 08028, Spain. J. Campos is with the Departament de Física, Universitat Autònoma de Barcelona, Bellaterra 08193, Spain.

Received 3 December 1993; revised manuscript received 18 April 1994.

0003-6935/94/296741-06\$06.00/0.

(C) 1994 Optical Society of America. generalization to any shape of the pixels and the pupil and draw some conclusions.

\section{Theoretical Background}

When a single Fresnel lens with focal length $f$ for a wavelength $\lambda$ is encoded in a pixelated low-resolution device (a quadratic phase is encoded), with a center-tocenter pixel distance given by $\Delta x$ and $\Delta y$, infinite new focal regions appear at the coordinates $(k X, l Y)$, where

$$
X=\frac{\lambda f}{\Delta x}, \quad Y=\frac{\lambda f}{\Delta y},
$$

and $(k, l)$ are arbitrary integers. $X Y$ also defines the apparent area of the lens associated with each focus. ${ }^{2}$ Then, if the device has $N \times M$ pixels with a rectangular pupil of dimensions $L_{x}=N \Delta x$ and $L_{y}=M \Delta y$, the number of lenses appearing in the device is given by

$$
W_{x}=\frac{L_{x}}{X}, \quad W_{y}=\frac{L_{y}}{Y},
$$

In Fig. 1 we can see a single binary LRFEL with $W_{x}=$ $W_{y}=3$. Note that it appears to be an array of $3 \times 3$ lenses.

The light distribution at a $(k, l)$ focus of an infinite phase-level LRFEL for a plane-wave illumination is given by [Eq. (20) of Ref. 2]

$$
\begin{aligned}
U_{k, l}\left(x_{i}, y_{i}\right)= & \frac{A}{i \lambda f} \frac{L_{x} L_{y}}{\Delta x \Delta y}\left\{\operatorname{sinc}\left(\frac{L_{x} x}{\lambda f}, \frac{L_{y} y}{\lambda f}\right)\right. \\
& \left.\times \exp \left[i \frac{2 \pi}{\lambda f}(x k X+y l Y)\right]\right\} \\
& * \operatorname{rect}\left(\frac{x}{\Delta x^{\prime}}, \frac{y}{\Delta y^{\prime}}\right),
\end{aligned}
$$




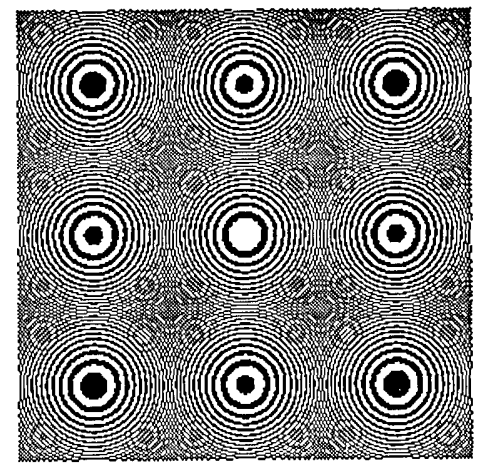

Fig. 1. Binary LRFEL with $W_{x}=W_{y}=3$; i.e., an array of $3 \times 3$.

where $A$ is the amplitude of the incident wave and * means convolution. The sinc function comes from the Fourier transform of the rectangular pupil of the device, and the rect function defines the pixel of dimensions $\Delta x^{\prime} \Delta y^{\prime}{ }^{4}$ Other useful parameters are

$$
c_{x}=\frac{\Delta x^{\prime}}{\Delta x^{\prime}}, \quad c_{y}=\frac{\Delta y^{\prime}}{\Delta y},
$$

In our development we take $A=1$ for simiplicity.

From Eq. (3) it can be deduced that there is a focal length that gives maximum intensity on the optical axis. If $c_{x}=c_{y}$ and $L_{x}=L_{y}$, then this maximum occurs for

$$
f_{\mathrm{opt}}=0.73 \frac{\Delta x^{\prime} L_{x}}{\lambda}
$$

We call $f_{\text {opt }}$ the optimum focal length because, giving a maximum intensity on the axis, the light distribution has a full width at half-maximum that is comparable with the width obtained with an infinite-resolution lens bounded by the same pupil.

\section{Diffraction Efficiency of the $(0,0)$ Focus}

From Eq. (3) the light-amplitude distribution for the $(0,0)$ focal region $\left(U_{0,0}\right)$ is given by

$$
\begin{aligned}
& U_{0,0}\left(x_{i}, y_{i}\right) \\
& \quad=\frac{1}{i \lambda f} \frac{L_{x} L_{y}}{\Delta x \Delta y} \operatorname{sinc}\left(\frac{L_{x} x}{\lambda f}, \frac{L_{y} y}{\lambda f}\right) * \operatorname{rect}\left(\frac{x}{\Delta x^{\prime}}, \frac{y}{\Delta y^{\prime}}\right) .
\end{aligned}
$$

To calculate the energy concentrated at the focus $E_{0,0}$, we must evaluate

$$
E_{0,0}=\int_{-\infty}^{\infty} \int_{-\infty}^{\infty}\left|U_{0,0}(x, y)\right|^{2} \mathrm{~d} x \mathrm{~d} y .
$$

Direct calculation of Eq. (7) is rather difficult. To simplify calculations, we can use the Parseval theorem, ${ }^{4}$ which establishes the possibility of evaluating $E_{0,0}$, by performing the surface integral on the square modulus of the Fourier transform of $U_{0,0}$. Making use of the Parseval theorem, we obtain

$$
\begin{aligned}
E_{0,0}= & \left(\lambda f c_{x} c_{y}\right)^{2} \int_{-\infty}^{\infty} \int_{-\infty}^{\infty} \operatorname{rect}\left(f_{x} \frac{\lambda f}{L_{x}}, f_{y} \frac{\lambda f}{L_{y}}\right) \\
& \times \operatorname{sinc}^{2}\left(\Delta x^{\prime} f_{x}, \Delta y^{\prime} f_{y}\right) \mathrm{d} f_{x} \mathrm{~d} f_{y},
\end{aligned}
$$

where $f_{x}$ and $f_{y}$ are the frequency coordinates in the Fourier space. This is the volume under the product of a squared sinc function and the rect function. In the general case it is the volume under the product of the Fourier transform of the pixel function (the function that defines the pixel) and the pupil function.

To study this expression, we define the following adimensional coordinates:

$$
g_{x}=\Delta x^{\prime} f_{x}, \quad g_{y}=\Delta y^{\prime} f_{y},
$$

and by using the definition of the rect function, we can rewrite Eq. (8) in terms of our parameters:

$$
\begin{aligned}
E_{0,0}= & L_{x} L_{y}\left(c_{x} c_{y}\right)^{2} \frac{1}{c} \frac{1}{d} \int_{-c / 2}^{c / 2} \int_{-d / 2}^{d / 2} \\
& \times \operatorname{sinc}^{2}\left(g_{x}, g_{y}\right) \mathrm{d} g_{x} \mathrm{~d} g_{y},
\end{aligned}
$$

where

$$
c=W_{x} c_{x}, \quad d=W_{y} c_{y} .
$$

We are encoding a phase function with ideal unity transmission. Then the amount of energy that goes through the LRFEL is

$$
E_{t}=L_{x} L_{y} c_{x} c_{y} .
$$

Thus we can define the diffraction efficiency $\epsilon_{t}$ as the ratio of the energy arriving at the $(0,0)$ focus $\left(E_{0,0}\right)$ and the total energy going through the lens $\left(E_{t}\right)$. Then $\epsilon_{t}$ is given by

$$
\epsilon_{t}=\frac{c_{x} c_{y}}{c d} \int_{-c / 2}^{c / 2} \int_{-d / 2}^{d / 2} \operatorname{sinc}^{2}\left(g_{x}, g_{y}\right) d g_{x} \mathrm{dg}_{y} .
$$

The total diffraction efficiency, taking into account the amount of energy illuminating the lens, is then given simply by $\epsilon=\epsilon_{t} c_{x} c_{y}$. We prefer to work with $\epsilon_{t}$, because in this way, $\epsilon_{t}=1$ then means that all the possible energy is concentrated at the $(0,0)$ order, and there is no concentration of light in other orders.

We can now study the behavior of this expression in terms of the values of the parameters $c$ and $d$. With Eqs. (10b), (4), (2), and (1) the relation of $c$ and $d$ to the focal length is

$$
c=\frac{L_{x} \Delta x^{\prime}}{\lambda} \frac{1}{f}, \quad d=\frac{L_{y} \Delta y^{\prime}}{\lambda} \frac{1}{f} .
$$




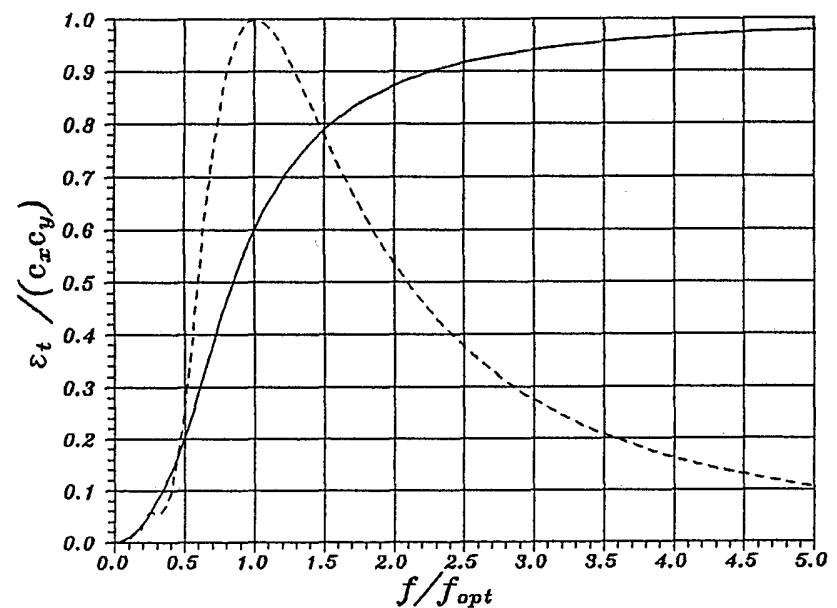

(a)

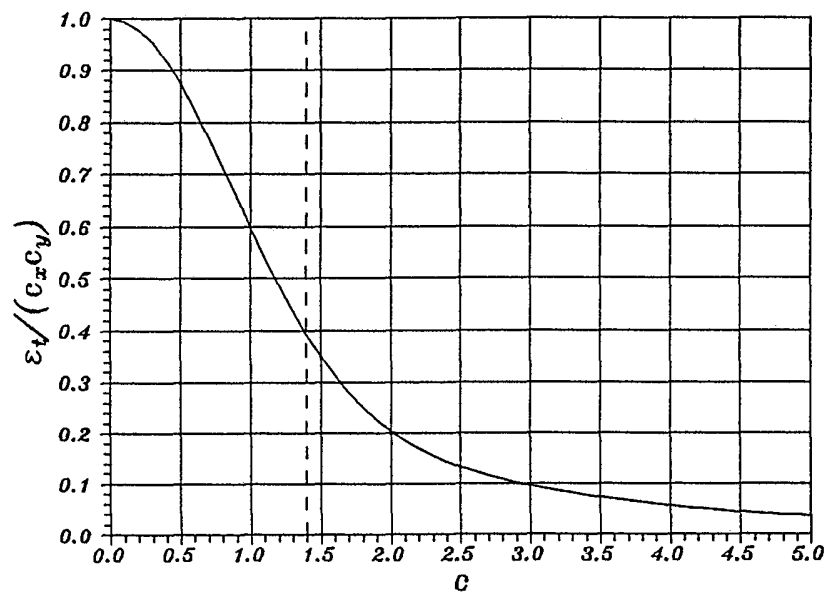

(b)

Fig. 2. Diffraction efficiency of the LRFEL at the $(0,0)$ focus as a function of the following: (a) the focal length normalized to $f_{\text {opt }}$ (the solid curve indicates efficiency and the dashed curve indicates the intensity on the optical axis); (b) the parameter $c$ (the dashed curve is for $c=1.34$ or $f=f_{\text {opt }}$ ).

We now study the following cases:

(i) A large focal length is encoded or there are small pixels: From Eq. (13), when $f \gg L_{x} \Delta x^{\prime} / \lambda$ and $f \gg L_{y} \Delta y^{\prime} / \lambda$, then $(\mathrm{c}, d) \ll 1$. In these conditions we say that a large focal length encoded. Then the value of the integral in Eq. (12) tends to the value of the product $c d$, and the diffraction efficiency is written simply as

$$
\epsilon_{t}=c_{x} c_{y} .
$$

Note that, if and only if $c_{x}=c_{y}=1$, the diffraction efficiency tends to unity. If not, the other $(n, m)$ focus will always concentrate a fraction of $E_{t}$, and the maximum diffraction efficiency available is less than one.

(ii) A short focal length is encoded: From Eq. (13), when $f \ll L_{x} \Delta x^{\prime} / \lambda$ and $f \ll L_{y} \Delta y^{\prime} / \lambda$, then $(c, d) \gg 1$. In these conditions we say that a short-focal-length-lens is encoded. The integral of

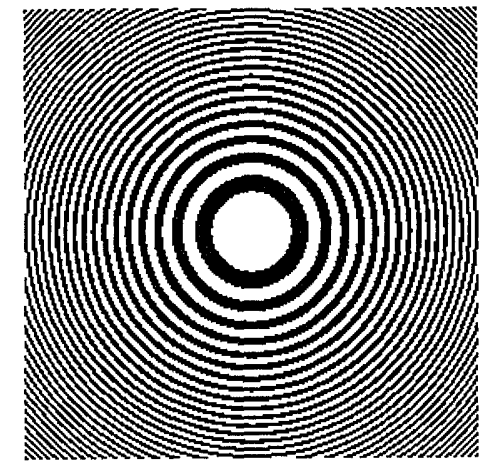

(a)

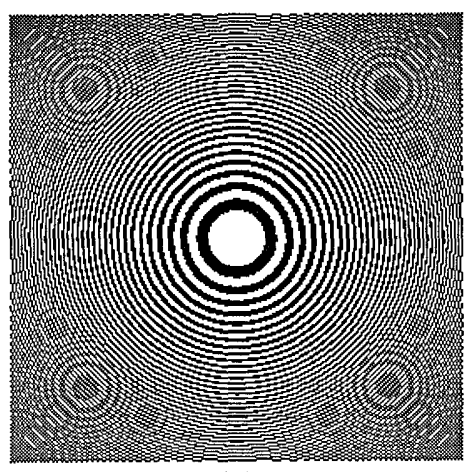

(b)

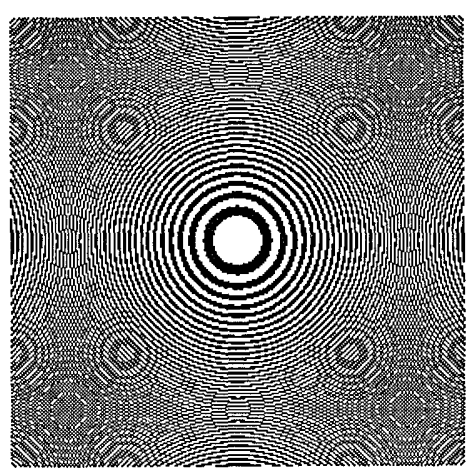

(c)

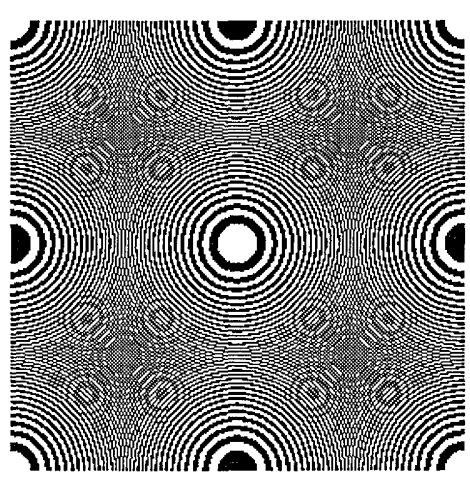

(d)

Fig. 3. Binary LRFEL corresponding to the following: (a) $c=$ $0.5,(\mathrm{~b}) c=1,(\mathrm{c})=1.34,(\mathrm{~d}) c=2$. From (a) to (b) there is a loss of $30 \%$ in the diffraction efficiency, from (b) to (c) there is a loss of $20 \%$, and from (c) to (d) is also a loss of $20 \%$. 
Eq. (12) tends to unity, and the diffraction efficiency takes the value $\epsilon_{t}=c_{x} c_{y} / c d$. This can be rewritten in terms of our parameters to obtain

$$
\epsilon_{t}=\frac{1}{W_{x} W_{y}}, \quad E_{0,0}=X Y c_{x} c_{y} .
$$

The value of $E_{0,0}$ means that the $(0,0)$ focus appears to concentrate only the light impinging on the transmitting apparent area of the $(0,0)$ lens.

(iii) The whole range of values of $f$ is included: This corresponds to the evaluation of Eq. (12) for all possible values of $c$ and $d$. The integral of Eq. (12) can be easily written as the product of two integrals. These integrals can be evaluated by standard numerical techniques. ${ }^{5}$ In Fig. 2 the diffraction efficiency $\epsilon_{t}$ divided by $\left(c_{x} c_{y}\right)$ is represented for the particular yet interesting case in which $c=d$; in this condition, from Eqs. (13) and (5), $f / f_{\text {opt }}=1.34(1 / c)$. The representation is given as a function of the focal length normalized to the optimal focal length $\left(f_{\text {opt }}\right)$ and the parameter $c$, respectively. The intensity on the axis is also represented in Fig. 2a. The diffraction efficiency increases with the encoded focal length encoded, and it falls very fast for short focal length; the maximum diffraction efficiency available is $\epsilon_{t, \max }=c_{x} c_{y}$. Note also that for the special case in which $c_{x}=c_{y}=1, c=W_{x}$ is the number of orders appearing. For $c=0.5, c=1, c=1.34$ (optimum focal length), and $c=2, \epsilon_{t} / c_{x} c_{y}$ is equal to $0.9,0.6,0.4$, and 0.2 , respectively. The lenses corresponding to these cases for $c_{x}=c_{y}=1$ are represented in Fig. 3 . Diffraction efficiency drops $30 \%$ between Figs. 3(a) and $3(b)$.

We can now study the focal length for which $c=0.5$ or, equivalently, $\epsilon_{t}=90 \% \epsilon_{t, \max }$ for the special case $c_{x}=c_{y}$ and $L_{x}=L_{y}$. Using Eq. (13) and $L_{x}=N \Delta x$, we can write $f_{90 \%}=2 N \Delta x^{2} c_{x} / \lambda$. In Table 1 we calculate $f_{90 \%}$ for some values of $\Delta x: \quad c_{x}$ equal to $1,0.75$, and $0.5 ; N=256 ; \lambda=632.8 \mathrm{~nm}$ (He-Ne laser). We can deduce that a focal length of $\sim 1 \mathrm{~m}$ with $c_{x}=1$ and $\epsilon_{t}=90 \%$ is available for $\Delta x<35 \mu \mathrm{m}$. If $c_{x}$ takes a smaller value, $f_{90 \%}$ is shorter; if $c_{x}=0.75$, then $\epsilon_{t}=$ $50 \%$; is $c_{x}=0.5$, then $\epsilon_{t}=22 \%$. High values of $c_{x}$ can be obtained with very-large-scale-integration techniques, but in a pixelated SLM, $c_{x} \approx 0.75$ is the maximum value available today. ${ }^{3,6}$

\section{Diffraction Efficiency for a $(k, l)$ Focus}

The light-amplitude distribution for the $(k, l)$ focal region is given in Eq. (3). Applying the same treatment as in the preceding case, we can write a similar
Table 1. Dependence of $f_{90 \%}$ on $\Delta x$ and $c_{x}$ for $N=256$ and $\lambda=632.8 \mathrm{~nm}$

\begin{tabular}{cccc}
\hline & \multicolumn{3}{c}{$f_{90 \%}(\mathrm{~m})^{a}$} \\
\cline { 2 - 4 }$\Delta x(\mu \mathrm{m})$ & $c_{x}=1$ & $c_{x}=0.75$ & $c_{x}=0.5$ \\
\hline 100 & 8.1 & 6.1 & 4.1 \\
50 & 2.0 & 1.5 & 1 \\
35 & 1.0 & 0.74 & 0.5 \\
25 & 0.5 & 0.37 & 0.25 \\
12 & 0.1 & 0.08 & 0.05 \\
\hline
\end{tabular}

${ }^{a}$ Proportional to $N$ and $c_{x}$.

expression for the diffraction efficiency:

$$
\begin{aligned}
\epsilon_{t}(k, l)= & \frac{c_{x} c_{y}}{c d} \int_{-c / 2}^{c / 2} \int_{-d / 2}^{d / 2} \\
& \times \operatorname{sinc}^{2}\left(g_{x}-k c_{x}, g_{y}-l c_{y}\right) \mathrm{d} g_{x} \mathrm{~d} g_{y} .
\end{aligned}
$$

In this case the sinc function is shifted $k c_{x}$ in the $x$ direction and $l c_{y}$ in the $y$ direction. In a general case the same shift will happen; we have the volume integral of the shifted Fourier transform of the pixel function multiplied by the pupil function. Let us now study some particular cases similar to those in Section 3.

(i) The lens is of short focal length: This implies that $c$ and $d$ are large numbers. Then if it is verified that $k c_{x}<c / 2$ and $l c_{y}<d / 2$, or equivalently, $k<$ $W_{x} / 2$ and $l<W_{y} / 2$, the maximum of the sinc function lies in the integration interval. These inequalities are equivalent to considering the orders that lie inside the pupil. From Eq. (16) the inequalities imply together with the short-focal-length condition that the value of the integral is near unity. Then, to a first rough approximation, we can say that all focal regions verifying these inequalities concentrate the same amount of energy with the diffraction efficiency given by Eq. (15).

It is easy to see from Eq. (16) that the order $k=$ floor $\left(W_{x} / 2\right) \equiv K$ and $l=$ floor $\left(W_{y} / 2\right) \equiv L$ [where floor $(x)$ represents the largest integer not greater than $x]$ is the least efficient verifying the inequalities because the sinc function lies near the corner of the integration interval. We can then calculate the diffraction efficiency for this order and the neighboring ones in order to obtain a lower limit for the diffraction efficiency of an array. We take as a reference $\epsilon_{t}(0,0)$, which is studied in Section 3. For calculations we take $k=K-n$ and $l=L-m$, where $n$ and $m$ are integers. Then we must evaluate

$$
\epsilon_{t, \mathrm{rel}}(K-n, L-m)=\frac{\int_{(-K-1 / 2) c_{x}}^{(K+1 / 2) c_{x}} \int_{(-L-1 / 2) c_{y}}^{(L+1 / 2) c_{y}} \operatorname{sinc}^{2}\left[g_{x}-(K-n) c_{x}, g_{y}-(L-m) c_{y}\right] \mathrm{d} g_{x} \mathrm{~d} g_{y}}{\int_{(-K-1 / 2) c_{x}}^{(K+1 / 2) c_{x}} \int_{(-L-1 / 2) c_{y}}^{(L+1 / 2) c_{y}} \operatorname{sinc}^{2}\left(g_{x}, g_{y}\right) \mathrm{d} g_{x} \mathrm{~d} g_{y}} .
$$


Making the changes

$$
g_{x}=g_{x}^{\prime}+(K-n) c_{x}, \quad g_{y}=g_{y}^{\prime}+(L-m) c_{y},
$$

in the upper integral, we obtain

$$
\begin{aligned}
& \epsilon_{t, \text { rel }}(K-n, L-m) \\
& =\frac{\int_{(-2 K+n-1 / 2) c_{x}}^{(n+1 / 2) c_{x}} \int_{(-2 L+m-1 / 2) c_{y}}^{(m+1 / 2) c_{y}} \operatorname{sinc}^{2}\left(g_{x}, g_{y}\right) \mathrm{dg}_{x} \mathrm{~d} g_{y}}{\int_{(-K-1 / 2) c_{x}}^{(K+1 / 2) c_{x}} \int_{(-L-1 / 2) c_{y}}^{(L+1 / 2) c_{y}} \operatorname{sinc}^{2}\left(g_{x}, g_{y}\right) \mathrm{d} g_{x} \mathrm{~d} g_{y}} .
\end{aligned}
$$

Note that $\epsilon_{t, \text { rel }}$ explicitly depends on $K$ and $L$. But if $K c_{x} \gg 1$ and $L c_{y} \gg 1$ (a large array is being encoded), this dependence disappears because the squared sinc function decays fast. For this particular case, $\epsilon_{t \text {,rel }}$ depends only on $n$ and $m$ because of its presence in the upper limit of the upper integral, and $\epsilon_{t}(0,0)$ is given in Eq. (15).

In Table 2 we have the values of $\epsilon_{t, \text { rel }}$ for $K=L, c_{x}=$ $c_{y}, k=1$, and $n=m$ for $K$ tending to infinite, and several values of $c_{x}$ and $n$. We can see that only the order $(K, K)$ has a relative efficiency lower than $90 \%$. Thus for encoding of a short focal length the LRFEL works as an excellent uniform array illuminator.

From Eq. (16), when $k c_{x}>c / 2$ or $l c_{y}>d / 2$ (this corresponds to noncodified orders $k>W_{x} / 2$ and $l>$ $W_{y} / 2$ ) the center of the sinc function lies outside of the integration interval (the main lobe contains $82 \%$ of the volume of the total sinc), and the concentrated energy in these orders is then reduced drastically. Note that for the special case in which $c_{x}$ and $c_{y}$ are small, an important portion of the main lobe of the sinc function may remain inside the integration interval, as can be deduced from Eq. (19) $(n, m<0)$. In this case the orders $(k, l)$ have a more gradual loss of energy when the values of $k$ and $l$ increase. This can be seen in Table 3 . We performed calculations for $k=0, l=N, N+1, N+2, N+3$, in the same conditions as in Table 2 . In this way we calculated the upper limit for the diffraction efficiency of the focal regions, verifying the inequalities for each condition.

(ii) A large focal length is encoded: In this case, $c$ and $d$ take small values. This implies an integration in the neighborhood of the coordinates' origin in Eq.

Table 2. Evolution of Diffraction Efficiency $\xi_{\text {, rel }}$ for the Higher Encoded Orders $(k, k)$

\begin{tabular}{cccc}
\hline & \multicolumn{3}{c}{$\epsilon_{t, \text { rel }}(\%)$} \\
\cline { 2 - 4 } Order & $c_{x}=1$ & $c_{x}=0.75$ & $c_{x}=0.5$ \\
\hline$l=k=K-0$ & 79 & 68 & 54 \\
$l=k=K-1$ & 93 & 91 & 90 \\
$l=k=K-2$ & 96 & 95 & 92 \\
$l=k=K-3$ & 97 & 96 & 95 \\
\hline
\end{tabular}

\begin{tabular}{cccc}
\hline & \multicolumn{3}{c}{$\epsilon_{t, \mathrm{rel}}(\%)$} \\
\cline { 2 - 4 } Order & $c_{x}=1$ & $c_{x}=0.75$ & $c_{x}=0.5$ \\
\hline$l=0, k=K-0$ & 87 & 82 & 73 \\
$l=0, k=K+1$ & 11 & 18 & 27 \\
$l=0, k=K+2$ & 3 & 5 & 6 \\
$l=0, k=K+3$ & 2 & 2 & 4 \\
\hline
\end{tabular}

(16). The integral can then be approximated by the product of the value of the sinc at the origin and the area of the integration interval:

$$
\begin{aligned}
\epsilon_{t}(k, l) & =\frac{1}{W_{x} W_{y}} \operatorname{sinc}^{2}\left(k c_{x}, l c_{y}\right), \\
E_{k, l} & =X Y c_{x} c_{y} \operatorname{sinc}^{2}\left(k c_{x}, l c_{y}\right) .
\end{aligned}
$$

Note that for the specific case in which $c_{x}=c_{y}=1$, the sinc function of Eq. (16) always take a zero at the origin of coordinates for any $(k, l)$ except $k=l=0$. In this case, practically all the energy is then concentrated in this order.

\section{Conclusions}

We have worked with the specific case of a device constituted by rectangular pixels and a rectangular pupil. This case is the most usual for a pixelated SLM, but this is a restriction that does not come from our development. As has been noted, replacement of the sinc function by the Fourier transform of a general pupil and the rect function by a general function defining the pixel in Eq. (3) leads to a completely analogous development. The only difference is that, in the general case, the resulting integrals for the diffraction-efficiency expressions are more difficult to evaluate.

Then, the diffraction efficiency of an LRFEL can be rigorously calculated from the light-amplitude distribution for every $(k, l)$ focal region. The amplitude distribution was obtained from a mathematical model of the LRFEL by application of the Fresnel diffraction approximation. The main results are as follows:

(i) If an LRFEL must be used as a single lens, a long focal length must be encoded. In this condition the maximum diffraction efficiency available, defined as the ratio of the energy impinging at the focus and the total energy going through the lens, is in general different from unity because the pixels (for technical reasons) are not as large as possible. Then other orders always concentrate a finite amount of energy.

(ii) The diffraction efficiency is a function of only two adimensional parameters depending on the characteristics of the device and the focal length encoded.

(iii) The diffraction efficiency is highly dependent on the focal length encoded. It always decreases as 
the focal length decreases. For the optimum focal length the diffraction efficiency is only $40 \%$ if $c_{x}=c_{y}=$ 1. If not, it is lower.

(iv) If an LRFEL is used as an array illuminator (a single LRFEL with a short focal length is encoded), the diffraction efficiency of all appearing lenses is approximately the same, except for higher orders, which have a smaller efficiency. For the nonappearing orders the concentrated energy is, in general, low.

This work was supported in part by Comision Interministerial de Ciencia y Tecnologia projects ROB91-0554 and TAP93-667-C01-01.

\section{References}

1. E. C. Tam, S. Zhou, and M. R. Feldman, "Spatial-light- modulator-based electro-optical imaging system," Appl. Opt. 31, 578-580 (1992).

2. E. Carcolé, J. Campos, and S. Bosch, "Diffraction theory of Fresnel lenses encoded in low-resolution devices," Appl. Opt. 33, 162-174 (1994).

3. D. M. Cottrell, J. A. Davis, T. Hedman, and R. A. Lilly, "Multiple imaging phase-encoded optical elements written as programmable spatial light modulators," Appl. Opt. 29, 25052509 (1990).

4. J. W. Goodman, Introduction a l'Optique Fourier et a l'Holographie (Masson, Paris, 1972), pp. 5-28.

5. W. H. Press, B. P. Flannery, S. A. Teukolsky, and W. T. Vetterling, Numerical Recipes in $C$, (Cambridge U. Press, Cambridge, 1990), pp. 111-141.

6. J. C. Kirsch, D. A. Gregory, M. W. Thie, and B. K. Jones, "Modulation characteristics of the Epson crystal television," Opt. Eng. 31, 963-970 (1992). 